\title{
Representaciones periodísticas de la pobreza en la prensa mexicana. Análisis
} de contenido*

\author{
Martín Echeverría Victoria** \\ Universidad ANáhuAC MAYAB
}

\begin{abstract}
Resumen: Las representaciones periodísticas de la pobreza pueden tener un efecto en la forma en que los lectores conciben esta problemática y valoran a sus sujetos; de ahí la importancia de un conocimiento objetivo de las mismas. Este trabajo expone resultados de un análisis de contenido de 1033 notas, provenientes de siete diarios de distintas entidades del país. Encontramos que tales representaciones son elaboradas con cierto profesionalismo, pero que colocan a los pobres en posiciones de dependencia del gobierno y las notas adolecen de superficialidad. Así, dichas representaciones son insuficientes para que los lectores conozcan y comprendan mejor dicha problemática. Palabras clave: Representaciones, prensa, pobreza, análisis de contenido, poder simbólico.
\end{abstract}

Abstract: The representations of the poor in the press can have an effect in how readers think about poverty and the poor themselves; therefore, it is important to produce objective knowledge about them. This paper

* El presente trabajo no hubiera sido posible sin la invaluable colaboración de los siguientes investigadores: Dr. Luis Gabriel Arango Pinto de la Universidad Simón Bolívar, Mtra. Alina García León, de la Universidad Regiomontana; Dra. Ada Gema Martínez Martínez, de la Universidad del Centro de México; Mtra. Beatriz Olivares Cruz de la Universidad Madero; Mtra. Mónica Savage Carmona, de la Universidad Intercontinental y Mtra. Lupita Trejo de la Universidad Vasco de Quiroga. Un agradecimiento también a los asistentes de investigación que trabajaron en este proyecto, y que por razones de espacio no podemos reconocer como se merecen.

** Comunicólogo. Maestro en comunicación por la Universidad Internacional de Andalucía, maestro en comunicación política por la Universitat Pompeu Fabra de Barcelona y candidato a doctor en Ciencias de la información por la Universidad de Sevilla. Profesor investigador de la Universidad Anáhuac Mayab. Sus líneas de investigación son los formatos audiovisuales de la comunicación política, y las representaciones mediáticas de los grupos vulnerables. echevemartin@yahoo.com.mx 
describes data from a content analysis of 1036 units, sampled from 7 papers edited in several regions across Mexico. We found that such representations are crafted with certain professionalism, but they put poor people as government dependent, and the news are superficial. In that way, such representations are not sufficient enough for the readers to know and understand poverty issues.

Keywords: Representations, press, poverty, content analysis, symbolic power.

A pesar de ser considerada la decimotercera economía más grande del mundo, en México la pobreza es uno de los problemas con mayor presencia y profundidad. De acuerdo con el Consejo Nacional de Evaluación de la Política de Desarrollo Social de México, en el año 2006, 44.7 millones de mexicanos vivían en condiciones de pobreza de patrimonio, $42.6 \%$ del total de habitantes del país, y 14.4 millones de mexicanos se encontraban en situación de pobreza alimentaria, $13.8 \%$. De estos, 5 millones se encuentran en zonas urbanas y 9.4 millones en zonas rurales. Según los índices de marginación, de 104359 localidades en el país $26.2 \%$ se encuentra en niveles muy altos de marginalidad, y $45.3 \%$ se encuentra en niveles altos (Coneval, 2009).

La pobreza tiene profundas raíces históricas relacionadas con las decisiones que las élites han tomado para conformar el modelo económico nacional (Valencia y Aguirre, 2001 en Gallardo \& Osorio, 2001). Consciente de las consecuencias de las transformaciones económicas sucesivas, el Estado ha implementado diversos programas dirigidos a medir y luego a enfrentar el rezago social. Hasta ahora sus resultados, como se nota escuetamente en las cifras, no son alentadores.

En México, la pobreza y los fenómenos de exclusión social relacionados con ella constituyen un problema antiguo. A pesar de que el bienestar es un compromiso constitucional y programático del régimen emanado de la Revolución de 1917, y de que todas las fuerzas políticas del país han coincidido en la necesidad de un sistema económico productivo capaz de proporcionar empleo, educación y salud a toda la población, no se ha podido construir un modelo económico estable capaz de atenuar la pobreza, extender la igualdad de oportunidades y generar un mínimo de bienestar para todos los habitantes. Las políticas asistencialistas y distributivas aplicadas por el Estado y por algunos actores sociales han tenido un éxito muy relativo en aliviar la pobreza, en tanto que las desigualdades generadoras de exclusión se han agravado (Gordon, 1997).

Al hablar de pobreza nos estamos refiriendo a un estado de carencia y de insatisfacción de necesidades básicas a tal grado que se traduce en una 
canasta básica insuficiente (pobreza alimentaria), privación de condiciones adecuadas de salud y educación (pobreza de capacidades) y carencias adicionales en vivienda, vestido y transporte (pobreza de patrimonio). (Coneval, 2009). De manera más amplia, la pobreza implica necesidades no satisfechas a un nivel psicosocial: Necesidades psicomorales - de autoestima, dignidad y autosuficiencia- y necesidades sociopolíticas (expresión pública, participación social, inclusión). (Leñero, 1995). De ahí que los pobres sean sujetos caracterizados por su vulnerabilidad: La fragilidad de sus condiciones de vida los hace proclives a situaciones de alto riesgo que implican un daño e imposibilitan el bienestar personal, tales como la prostitución, la drogadicción, la violencia, la delincuencia, la migración, entre otros. Fundamentalmente la pobreza es una condición de exclusiones (sociales, políticas y culturales) que deriva en una participación desigual en el ejercicio de la ciudadanía - lo que remite a lo indigno de esta condición. En consecuencia, la pobreza vulnera los derechos elementales de los sujetos porque los pone en situación potencial de abuso (de las autoridades, de otros estratos sociales, de las instituciones privadas o públicas, etcétera) lo que agudiza su severidad y ayuda a reproducirla.

De la gravedad del problema — en extensión y profundidad - se desprende la expectativa legítima de que los medios de comunicación y, particularmente la prensa, lleven a sus páginas una de las principales preocupaciones del país y procuren el entendimiento, por parte de la sociedad, de sus particularidades. ¿Por qué nos importa cómo se comunica la pobreza en la prensa diaria? Por su profundidad, amplitud y complejidad, la pobreza constituye un fenómeno que es conocido por la sociedad primordialmente a través de representaciones: ya sea en forma de reportes estadísticos, informes sociológicos o antropológicos o reportes de prensa, las discusiones sobre la misma tienen como soporte y referente las representaciones sociales que determinadas instituciones — políticas, eclesiásticas, mediáticas- elaboran. En función de esta modalidad de cognición social y del control que las instituciones mediáticas ejercen sobre ella, la cobertura periodística de la pobreza tiene significativas repercusiones sociales; en primer lugar, porque las representaciones liberadas al espacio público alimentan las cogniciones, creencias y valoraciones que diversos grupos sociales sostienen sobre ese fenómeno, al grado de contribuir a la difusión, modulación o creación de los estereotipos dominantes de este grupo en la sociedad (Richardson, 2007). Y en segundo lugar es factible suponer que ciertas actitudes y pautas de acción —o inacción- que se manifiestan hacia dicho segmento social están influidas por esas cogniciones (Vasilachis, 2003). Así, una representación negativa de la 
pobreza puede, en el peor de los casos, reforzar las prácticas de exclusión existentes en la sociedad, al justificarlas - como en las representaciones asociadas al crimen o a la pasividad - y consolidar en consecuencia las prácticas asociadas al clasismo (Golding, 1999). En contraparte, las representaciones realistas y completas pueden ayudar a promover una conciencia social sobre la pobreza que parta de un principio de igualdad y desde ahí derive en otros valores de inclusión y de respeto a los modos de vida de los otros, así como incrementar nuestra capacidad de empatía y solidaridad hacia ese grupo (FSP, 2007).

Lo que exponemos en este trabajo es, en síntesis, una descripción sistemática y objetiva de las representaciones de la pobreza en una parte representativa de la prensa mexicana, elaborada a partir de una exploración cuantitativa extensa en el tiempo y en el espacio territorial, realizado a través del método de análisis de contenido. La extensión territorial se consiguió con la inclusión de investigadores de distintos estados del país que recopilamos los datos correspondientes a rotativos locales. La extensión temporal se logró analizando información periodística publicada en los últimos cinco años; la determinación de un lustro nos parece adecuada para una retrospectiva de peso sobre este tema en un lapso histórico relativamente significativo, y proporciona una base sólida para hacer inferencias plausibles sobre las representaciones de la pobreza que posiblemente se hayan sedimentado en los lectores de estos medios, así como acerca del comportamiento de los emisores. De manera más detallada la realización del estudio se propuso, a su vez: 1 ) determinar la relevancia que el tema de la pobreza tiene para los medios impresos mediante la cuantificación de las notas que los periódicos destinan a la cobertura de acontecimientos relacionados a dicho tema y su topografía; 2) describir los atributos de representación de las personas en situación de pobreza; 3 ) describir los contextos sociales en medio de los cuales se representan los acontecimientos relacionados a la pobreza, los actores relacionados con la misma, así como su tipo y grado de relación con la problemática y; 4) identificar las prácticas periodísticas que se infieren en la cobertura de la pobreza. De manera complementaria evaluamos los datos de las notas en relación con la magnitud del fenómeno de la pobreza en México, en el sentido de si éstas permiten a los lectores un conocimiento adecuado del tema en su entorno nacional.

Finalmente, pensamos que esta investigación puede ser el precedente de una herramienta de veeduría mediática destinada a la medición continua de los indicadores aquí propuestos, y de otros instrumentos aplicados a la indagación acerca de los diversos grupos vulnerables que están en una situación similar en la estructura social. 


\section{PREMISAS TEÓRICAS}

En otro trabajo (Echeverría, 2007) exponemos de manera extensa las consideraciones teóricas que creemos adecuadas para abordar este tema, por lo que mencionamos brevemente las siguientes premisas: 1) El periodismo se concibe como un dispositivo de ejercicio de poder simbólico, que tiene la capacidad de difundir ampliamente representaciones objetivas a través de las cuales se define la realidad social. (Bourdieu, 2000; Couldry, 2000) Tales representaciones son eficaces porque constituyen un conocimiento externalizado que, mediado por el lenguaje, es interiorizado por los sujetos y validado a su vez como objetivo (P. Berger \& Luckmann, 2005). El periodismo como modalidad de concentración de poder simbólico tiene la particularidad de estar conducido por agentes de capital que poseen los medios de producción de información, controlan el conocimiento colectivo, y participan de un orden social desigual, de concentración y distribución fuertemente inequitativa de la riqueza (Kendall, 2005; Martín Serrano, 1994). En consecuencia, están interesados en preservar dicho status quo modulando las formas en que éste se representa y atenuando la precariedad económica derivada del mismo (Murdock \& Golding, 1982; Wayne, 2003). De ahí que la representación interesada del fenómeno de la pobreza sea una manifestación de la capacidad del poder simbólico, y sus agentes, para excluir a este segmento social de los discursos de los medios y por ende, de la participación y el reconocimiento social. 2). Más allá de estos problemas estructurales, los periodistas están acotados por constricciones económicas, organizacionales e ideológicas que condicionan la manera en que comunican la pobreza. Actúan bajo criterios de maximización de recursos, ante lo cual la "calidad" se ve reñida con la "contabilidad" de la empresa periodística (Golding \& Murdock, 2000). Se desempeñan en una organización diseñada para dar cuenta, a una gran velocidad, de tipificaciones de acontecimientos (no sucesos espontáneos) para lo cual dependen de la estabilidad de fuentes que les provean de información preelaborada y adecuada a su ritmo de producción (Tuchman, 1983). Por último, pertenecen a una clase social con sus propios intereses y esquemas culturales identificados con los valores dominantes del sistema capitalista (Maigret, 2005) 3). En consecuencia, y de acuerdo con diversos autores, se manifiestan ciertas operaciones discursivas tendientes a representar a la pobreza como: $a$ ) un problema individual, voluntarioso, atribuible no al sistema que lo origina sino a la incapacidad del sujeto de adaptarse a él en virtud de cierto desajuste ante el orden social (Golding, 1999); $b$ ) un tema que 
emerge en la medida en que el acontecimiento pueda "espectaculizarse", es decir, cuando los acontecimientos narrados puedan asumir valores de entretenimiento - como en las tragedias o desastres naturalesSampedro \& Codeiro, 2001) y; c) cuya visibilidad es dependiente de la voluntad de otros actores, tales como las élites políticas y económicas, de "ponerlo en escena" (Vasilachis, 2003). Todo esto lo coloca como un tema de cobertura, mínima, contingente — no está tematizado- y en consecuencia simplificado. 4). A propósito de las anteriores premisas, se han encontrado ciertos encuadres característicos que el periodismo elabora ante la pobreza: Victimización (negación de la capacidad de agencia de los sujetos en pobreza, lo que implica dependencia a otros grupos sociales), desindividualización (la pobreza se homogeneiza, sus contextos desaparecen), reduccionismo (se reduce a cifras y datos estadísticos) y desviación social (las regiones pobres como "zonas del mal") son los más recurrentes (G. Berger, 2003; Gamson, Croteau, Hoynes \& Sasson, 1992; Rey, 2004; Vasilachis, 2003). Las dos primeras premisas pudieran considerarse factores de explicación de la elección temática y el comportamiento de la prensa respecto a este tema. En el caso de las dos últimas, corroboramos en el terreno empírico en qué medida se confirman o, en dado caso, se matizan.

\section{Metodología}

Siete investigadores pertenecientes a universidades de los estados de Yucatán, Puebla, Michoacán, Monterrey, San Luis Potosí y el Distrito Federal, seleccionaron cada uno un periódico de referencia de su localidad para analizar. El corpus fue integrado mediante el método de muestreo estratificado denominado semana compuesta, que consiste en la construcción de "semanas tipo" que representan el contenido publicado en un determinado lapso por un medio impreso. La población de este estudio consistió en todos los ejemplares que un determinado periódico publicó en los cinco años previos (2008, 2007, 2006, 2005, 2004), mientras que la muestra fue conformada, de acuerdo con el método descrito, por los ejemplares contemplados en las 10 semanas construidas (considerando dos semanas por año). Por lo tanto, la muestra se integró de los 70 ejemplares que un determinado periódico publicó en 5 años previos, que representa confiablemente a todos los ejemplares publicados en ese periodo de tiempo.

La unidad de estudio fue la nota - se excluyeron deliberadamente los géneros de opinión- y su identificación se hizo bajo tres criterios 
de selección: Cuando se reportaran temas específicos relacionados con la precariedad social, cuando se manifestaran sujetos caracterizados como pobres bajo determinados atributos y; cuando se informara de las actividades de organizaciones altruistas o asistencialistas de los grupos en pobreza. Entre los siete rotativos estudiados se integró un corpus de 1033 unidades en total.

Las notas fueron analizadas con un protocolo diseñado a partir de estudios precedentes (Fundación para la Superación de la Pobreza, 2007; Golding, 1999; Hernández, 2003; Kendall, 2005; Rey, 2004; Tablante, 2006; Vasilachis, 2003) y cuya aplicación pasó por tres pruebas simples de confiabilidad. Las variables utilizadas en este protocolo se aglutinan en cuatro secciones que corresponden a cada una las preguntas de investigación elaboradas en este trabajo. Una primera sección comprende la topografía y la magnitud de la cobertura de la pobreza; ahí se tomaron en cuenta las variables de sección de la nota, número de página, tamaño de la nota y tipo de nota - directamente o indirectamente asociada a la pobreza. Una segunda sección intenta conocer las características sociodemográficas de las personas en pobreza. Se implementaron para ello las variables de género, etapa de vida, ocupación, condiciones sociales especiales que merezcan una mayor cobertura — discapacidad, enfermedad o pertenencia a etnias-, el encuadre o concepción del fenómeno, así como la dimensión predominante de la pobreza, es decir, la carencia central a partir de la cual se narra el acontecimiento - carencias materiales, de salud o cultura, prácticas de exclusión, conflicto familiar o social y limitaciones en el desarrollo de capacidades- determinadas a partir de las mediciones oficiales de la pobreza (Coneval, 2009) y otros trabajos (Ortega Villa, 2007). La tercera sección mide los contextos sociales en los que se manifiesta la pobreza, lo que da pie a conocer qué acontecimientos e instituciones favorecen la cobertura del tema y qué tan dependiente es ésta a los mismos; aquí se incluyen las variables de tema —utilizando una categorización previa de 33 de ellos-, los ámbitos geográficos donde se representan y el grado de agencia de las personas en pobreza que participan en acciones que implican su superación. La sección final indaga acerca de las decisiones editoriales que los periodistas toman para cubrir el tema y representar al referente. Medimos la materialidad de la información en las notas - cifras, acontecimientos o discursos-, el género informativo, la modalidad proactiva o reactiva de cobertura — bajo ciertos indicadores - el tono de la nota, el número y tipo de fuentes consultadas para elaborarla, el uso de fotografías en la cobertura así como los referentes contenidos en ellos. 
Exponemos los resultados significativos del análisis a partir de las secciones expuestas anteriormente:

a) Topografía y magnitud de la cobertura de la pobreza. Las notas relacionadas con el tema de la pobreza tienen una presencia discreta en los medios de estudio. Esto lo afirmamos basándonos en dos hallazgos: En primer lugar, en cuanto a la visibilidad del tema en las páginas de los periódicos (figura 1), puesto que la página promedio en donde se coloca la información es la 12, de visibilidad baja — aunque la desviación estándar de 11.4 implica que el tema se dispersa considerablemente más allá de este promedio. Incluso cuando dividimos las posiciones en cohortes de visibilidad, tenemos que sólo $9.2 \%$ de las notas tiene visibilidad plena, y $44.1 \%$ tiene visibilidad baja. Un segundo hallazgo es en cuanto al tamaño que ocupan las notas, que tiende a ser reducido: $45.4 \%$ de las mismas ocupan menos de un cuarto de plana de tamaño, $30.4 \%$ ocupa un cuarto de plana y el sólo $13.4 \%$ ocupa media plana.

Desde el punto de vista de la topografía, el hecho de que la mayor parte de la información se encuentre en la sección local (46.9\% de las notas, comparado con $26.9 \%$ de la sección nacional y sólo $10.2 \%$ internacional) implica que la información generada acerca del tema tiene rasgos de proximidad e inmediatez, lo que le permite al lector conocer en primera instancia la pobreza en su entorno y luego en espacios geográficos remotos. A pesar de esta adecuada posición, a final de cuentas nuestras cifras indican que la pobreza no está tematizada en los medios, es decir, no concita la atención y cobertura sostenidas de los periodistas: sólo $30.4 \%$ de las notas trataron específicamente del tema de la pobreza, mientras $69.6 \%$ restante trataron otros temas a los que la pobreza se asocia solamente. Tenemos, pues, una presencia discreta del tema de la pobreza, en términos cuantitativos de topografía, colocada y elaborada en gran medida en el espacio de lo local, que es más próximo, pero que cualitativamente tiene menos peso porque es enunciado de manera tangencial a otros temas. Dicha caracterización confirma lo expuesto en el cuerpo teórico en el sentido de que la cobertura de la pobreza es contingente, es decir, se activa principalmente al momento en que las élites lo evocan u otros temas lo hacen emerger, y que por lo tanto, su cobertura pudiera tener un carácter reactivo, no intencionado. Sin embargo no podemos afirmar que el tema merece una mínima cobertura por parte de la prensa: Si bien el fenómeno no está tematizado, es decir, no forma parte de la agenda de los rotativos porque es infrecuente que éstos le confieran visibilidad, existen frecuencias que implican cierta visibilidad 
intermitente: $9.2 \%$ de visibilidad plena de las notas o $13.4 \%$ de las mismas ocupando media plana significa que en momentos excepcionales, posiblemente relacionados con acciones de gobierno o acontecimientos trágicos, el tema se manifiesta con fuerza, lo que le impide desaparecer.

b) La representación de las personas pobres. Las características demográficas cuantificadas permiten una lectura doble: Acerca de los rasgos específicos cuantificados y adicionalmente a su sola presencia o ausencia, que en ciertos casos es más significativa. Es el caso de las mediciones acerca del género de los sujetos representados, su etapa de vida o rol laboral. En dichas variables la mitad de la notas no tienen identificación $(62.1 \%$ para género, $61 \%$ para etapa de vida y $73.3 \%$ para el rol laboral), la representación abstracta y generalizada ("los pobres"), predomina sobre la casuística. Esto se acentúa ante el hecho de que la caracterización de los sujetos, cuando sí se proporciona (como en el caso de los roles), está colocada en zonas de baja visibilidad: Los trabajadores manuales, por ejemplo, merecen 74 notas en páginas de baja visibilidad y sólo 15 en primera plana. Esto ocurre también al momento de expresar si la situación de pobreza se relaciona con factores individuales o sistémicos. En más de la mitad de las notas (58.6\%) no se identifica ninguno de los dos encuadres, síntoma también de que los datos que expresan una mínima causalidad son infrecuentes. Más allá del significado de estas omisiones, el primer fenómeno implica que a nivel identitario el atributo de pobreza está jerárquicamente por encima de los otros atributos sociales, e implica de paso una cierta debilidad profesional por parte del periodismo al no construir una casuística más referenciada de los sujetos de los que informa, y no proporcionar los datos suficientes que le permitan al lector ubicar las afectaciones sensibles que los acontecimientos tienen en las personas. La no especificación del género del representado es un caso ejemplar. Puesto que la mujer es quien padece con mayor agudeza las consecuencias de la pobreza (Riquer, 2002), la atenuación de este hecho por parte de la prensa (16.7\% de los representados son mujeres, $21.2 \%$ son hombres) impide que los lectores le den este importante matiz. Las frecuencias indican que los criterios de cobertura reproducen ciertas veces algunos estereotipos recurrentes acerca de la pobreza. No lo hacen en el caso de los indígenas o las personas con discapacidad porque estos segmentos sociales aparecen con muy poca frecuencia ( $13.8 \%$ y $1.6 \%$, respectivamente), manifestando de paso que la exclusión simbólica a la que están sometidos es incluso más aguda que la de los sujetos en pobreza. Pero sí lo hacen en el caso de los roles laborales en donde predomina la presencia de campesinos (8.6\%) y obreros $(1.5 \%)$, desvinculando así a otras ocupaciones del impacto de la pobreza. 
Al condensar los roles laborales en tipologías de trabajo, sobresale que la mayor parte de las ocupaciones relacionadas con la pobreza son de tipo manual (49.5\%), dedicadas a la producción de bienes. A los trabajadores de alguna forma de provisión de servicios (8.4\%) o comercio (6.5\%) -incluso en ocupaciones como el ambulantaje - se les identifica mucho menos con el fenómeno de la pobreza, puesto que su mención es infrecuente en los rotativos. Es manifiesta asimismo la simplificación del tratamiento de la pobreza a partir de las reducidas dimensiones con las que se describe. La concepción tradicional de la pobreza como un estado de carencia de satisfactores materiales (comida: 9.3\%; techo: $12.1 \%$ y; otras carencias en general: $6.2 \%$ ) se reproduce aquí; sumadas las variables que conforman una dimensión material del problema, tenemos que en $61.4 \%$ de las notas ésta es la principal caracterización, frente $12.7 \%$ de las notas en que se caracteriza como un problema de exclusión social, 9.3\% asociado a la falta de desarrollo de capacidades, $8.1 \%$ como formas de conflicto familiar o social, o $7.7 \%$ como un tema relacionado con la vulnerabilidad en salud. Tal centralidad contribuye por su parte a invisibilizar o atenuar las múltiples consecuencias que el fenómeno tiene en los sujetos.

Como agravante tenemos que dicha dimensión es la más visual y en ese sentido "espectacularizada": $41.8 \%$ de las notas se acompaña con una fotografía de sujetos en pobreza. Como parte de una caracterización general y vaga de los sujetos pobres, se opta por darle a la pobreza una causalidad predominantemente sistémica (atribuible a la estructura, en 35.5\% de las veces) y muy pocas veces individualista (relativa a la inadecuación, sobre todo moral, del individuo, en 5.9\% de las notas). Independientemente de si este tratamiento es adecuado para evitar estigmatizaciones individuales o inadecuado por el potencial de victimización que pueda producir, los datos nos guían a suponer que más que una comprensión intencionalmente solidaria de la pobreza con sus sujetos, hay en cambio una visión poco subjetiva de la misma. Así, la tesis expuesta al principio de este trabajo acerca de la caracterización desindividualizada de la pobreza se verifica mediante este análisis: Las caracterizaciones y causalidades son generalizadoras y abstractas, el tratamiento de la pobreza sigue los patrones estereotípicos que acotan su comprensión cabal.

c) Contextos y actores sociales relacionados con la cobertura de la pobreza. Por otro lado, los contextos en donde se manifiesta el tema de la pobreza están caracterizados por la presencia de élites gubernamentales (en $19.8 \%$ de las notas se tratan temas relacionados a la atención estatal de la pobreza) y el fenómeno de la migración (8.4\%). Sin embargo, también figuran temas relacionados con afectaciones de los sujetos en pobreza, tales como problemas de vivienda (5.2\%) y alimentación (5.1\%). De 
manera sobresaliente en $5.3 \%$ de las notas los pobres son representados desempeñando acciones a favor de sus propias causas (de vivienda, derechos civiles o apoyos gubernamentales, entre otros). La preeminencia de temas relacionados con las élites gubernamentales, patentado en la frecuencia de notas (que duplica al tema que le sigue) y el tamaño de las mismas $(18.4 \%$ de las notas con temas gubernamentales ocuparon una plana entera, frente $7.9 \%$ de las notas referentes a la migración), habla del grado de institucionalización del periodismo mexicano y del poder de dichas élites para influir en la agenda mediática y capturar temas como éste. También es, de forma cosustancial, un indicador de la dependencia del propio periodismo hacia estas entidades, particularmente en su calidad de fuentes (vid infra). Sin embargo, no podemos desconocer la atención prestada por los rotativos a las actividades de los propios pobres que, aunque en un porcentaje varias veces inferior al Estado, se constituye como una ventana de visibilidad de cierto valor. Por otra parte, en más de la mitad (55.7\%) de las notas se entabla una relación entre un agente y los sujetos en pobreza, dirigida a superar sus precarias condiciones; ahora bien, en $35.7 \%$ de las notas las personas en pobreza fungen como receptores de la ayuda de dichos agentes, frente a $14 \%$ que actúan como gestores y $5.9 \%$ como co-gestores. Esta dependencia se concentra casi en su totalidad en la dimensión más visible de la pobreza, esto es, la carencia de recursos materiales $(47.4 \%$ de las notas alude a dicha dimensión, en contraste con $12.5 \%$ que le sigue, que habla sobre problemas de salud), aunque en general en cualquier dimensión del fenómeno en el que participen determinadas élites sociales (particularmente gubernamentales), los sujetos en pobreza se representan como receptores de la ayuda de las mismas. Desde el punto de vista político tal caracterización implica que los pobres son sujetos pasivos a ser atendidos primordialmente en las afectaciones inmediatas y visibles de su condición, y que la responsabilidad del gobierno hacia la pobreza inicia (y posiblemente acabe) en la satisfacción prioritaria de dichas necesidades en detrimento de otras que también surgen como consecuencia de la precariedad. Así, estos contextos de emergencia manifiestan que, en efecto, la pobreza se cubre preferentemente por la prensa al escenificarse, es decir, en el contexto de una puesta en escena por parte de las élites mencionadas en virtud de sus intereses específicos. Tal móvil proporciona mayores elementos para sostener que los rotativos son reactivos a la hora de cubrir el tema de la pobreza.

Al no tener fuentes institucionales que solventen los costos de cobertura, se produce un vacío de conocimiento de la pobreza por parte de los periodistas, más que un efecto de distorsión activa. De ahí que, llenando tal vacío, se reproducen selecciones y expresiones temáticas, contextuales o 
caracterológicas cercanas al sentido común de la clase media: El sujeto pobre promedio es un campesino u obrero con necesidades de comida, vestido y techo, afectado duramente por las condiciones económicas que lo oprimen, y dependiente del paternalismo del gobierno. No hay un énfasis particular en el género ni en la etapa de vida, porque el sentido común no penetra con tanto detalle en dichas especificidades.

d) Prácticas periodísticas de cobertura de la pobreza. La jerarquía que un tema ocupa en el rango de intereses de los periodistas tiene una manifestación material - la nota - a través de la cual es posible inferirla. El análisis extensivo de esta materialidad nos permite afirmar que la cobertura del tema de la pobreza moviliza pocos recursos periodísticos: La información es superficial, generada de manera pasiva (por iniciativa de la fuente y no del periodista), el número de fuentes es limitado y se trata de fuentes cuya colaboración con la prensa es naturalmente estrecha, pues proveen de información de manera rutinaria y a bajo costo. En contraparte, el tono en general no es sensacionalista, la nota se elabora a partir de acontecimientos y no datos secundarios, y la información gráfica, tan susceptible a dramatizar el acontecimiento, se maneja con prudencia. La información es superficial porque la amplia mayoría de unidades de información se trata de notas breves (79.3\%), en lugar del reportaje (9.9\%) o la crónica (6.5\%), géneros más apropiados para comprender mejor un tema de la complejidad de la pobreza. Es pasiva porque la medición acerca de la iniciativa de cobertura por parte del reportero es reducida (sólo $25.7 \%$ de las notas se elaboraron de manera proactiva). Aunado a ello, en una minoría de notas que tratan de la pobreza se consulta a más de una sola fuente al cubrirla (15.9\% tiene dos fuentes, $10.3 \%$ tres fuentes y $4.4 \%$ cuatro fuentes; $56 \%$ de las notas se elabora con una sola fuente). El poder de las élites, particularmente del Estado, para fungir como fuente (lo es en $40.5 \%$ de las notas) y construir así el acontecimiento, presenta un agudo desequilibrio respecto a los sujetos en pobreza que son fuentes para los medios (en $22.1 \%$ de las notas desempeñan ese papel), aunque no tanto como en el caso de los expertos (11.4\% de las notas) y la sociedad civil (10.6\%). La prensa no recoge la experiencia de la pobreza en voz de sus propios sujetos, no recurre a actores que la puedan explicar con mayor profundidad (expertos) o interés (sociedad civil) y sí acude, en cambio, a las fuentes solícitas del Estado, con las que trabaja cotidianamente y que proveen información de bajo costo, prácticamente subsidiada, como insumo para la nota.

Un tema al que se le dedican reportajes, en el que participan numerosas fuentes por unidad de información y que involucra el trabajo proactivo del periodista para salir a buscar fuentes de información distintas de las que 
tiene a la mano, es un tema que tiene prioridad en la agenda del medio, razón por la cual se le dedican todos esos recursos a su cobertura. Claramente el tema de la pobreza no figura en las prioridades de cobertura de los medios analizados. Pero a pesar de una jerarquía baja en los intereses de los rotativos, existen elementos para caracterizar una conducción responsable del tema (entendida como acorde a los lineamientos normativos del periodismo liberal): la mayoría de las notas tienen como materia prima al acontecimiento (54.4\%), y no al discurso público (32.6\%) o a las cifras sobre el fenómeno (13\%). Esto sugiere un cierto grado de elaboración de la nota, además de que permite un conocimiento más sensible de la pobreza por parte del lector. Además prueba que no se manifiesta el reduccionismo económico del tema, como expusimos en el cuerpo teórico. La poca profundidad de las notas se comunica ciertamente con criterios neutrales de narrativización (79.7\% de las notas), dejando de lado la dramatización (15.2\%) y el sensacionalismo (3.3\%) en el relato acerca de la pobreza. Una señal más de responsabilidad periodística es respecto a las decisiones en cuanto a información gráfica. El porcentaje de notas en donde se fotografía únicamente a personas en pobreza es relativamente reducido (34.1\%) Los desastres naturales y las tragedias no merecen una cobertura fotográfica completa (en $40.9 \%$ de las notas acerca de estos acontecimientos se retratan los sujetos en pobreza involucrados); considerando la rentabilidad visual de los sujetos pobres en medio de desgracias o simplemente en la cotidianidad de su miseria, observamos frecuencias moderadas que sugieren que un criterio editorial de cierto nivel de profesionalismo ejerció un control sobre estos posibles excesos.

En síntesis, la contingencia de la cobertura de la pobreza se manifiesta a la luz de estos indicadores, al permitir observar más de cerca el carácter reactivo con el que se produce la nota y en general la limitación de recursos dedicados a su cobertura, que implican así una forma de exclusión, ahora simbólica, de este grupo. Se presenta, no obstante, la aplicación de ciertos parámetros de profesionalismo periodístico en virtud del carácter "de referencia" de estos medios, que prescriben un tratamiento considerablemente neutral del tema.

e) Evaluación de los datos respecto al referente. El asunto de si la cobertura está a la altura de la extensión y profundidad del referente, se sanciona en virtud del contraste entre dos agendas, la mediática y la social. En lo social, como afirmamos anteriormente mediante datos duros, la magnitud del fenómeno de la pobreza en México y la hondura de sus condiciones lo colocan naturalmente en la cima de los temas sociales prioritarios, aún cuando su vasta complejidad y lo remoto de su solución provoque distanciamientos esporádicos hacia el mismo por parte de la 
sociedad. La agenda mediática no tiene en cambio este matiz de urgencia o relevancia, porque la pobreza no está tematizada en sus páginas, ocupa mayoritariamente zonas de baja visibilidad, reducido tamaño y, sobre todo, la elaboración de la nota no es proactiva, tiene pocas fuentes y la caracterización de los sujetos pobres es abstracta: En pocas palabras, el medio no moviliza los recursos periodísticos que la relevancia social del tema merece. Sospechamos que de incluirse la pobreza con fuerza en la agenda gubernamental, y tomando en cuenta la relativa porosidad de los medios impresos a aquélla, el grado de relevancia sería distinto.

Representaciones periodísticas de la pobreza en la prensa mexicana. Palabra finales. Desde un punto de vista macrosocial, atestiguamos el ejercicio del poder simbólico actuando en la atenuación de las representaciones públicas que revelan la desigualdad social de un determinado entorno. Tal atenuación opera con mayor fuerza en el caso de grupos desempoderados, como los sujetos en pobreza, que desprovistos de capital económico y cultural tienen acceso restringido o nulo al proceso de construcción de dichas representaciones. El fenómeno descrito tiene potenciales consecuencias en el entorno social.

Para la clase media que consume los rotativos analizados, posiblemente en la realidad social conocida a través de los medios analizados, la pobreza no tiene una presencia constante, sus características específicas son unívocas cuando no vagas, y las élites sociales asisten con frecuencia a su rescate. No obstante, no se leen en los diarios explorados aspectos suficientes que produzcan un efecto de estigma hacia los sujetos en pobreza. Las características específicas de este dispositivo de poder simbólico, el periodismo, son claves para comprender este fenómeno: Por un lado, un sistema de mercado que ejerce fuertes constricciones a la elaboración de la nota; por otro, las rutinas periodísticas y organizacionales preocupadas por producir el acontecimiento con inmediatez y en las fuentes más asequibles.

Finalmente la falta de familiarización y sensibilidad hacia el tema de parte de los productores de la noticia, los periodistas, individuos educados de clase media cuyo contacto con otros segmentos sociales está más orientado, por norma profesional, a las élites. Mención aparte merece el papel de una ideología que es transversal a estos sistemas y que favorece la reproducción del status quo de desigualdad económica y concentración de la riqueza. Así, la presencia de tales condicionamientos concebidos como fuerzas revela la contradicción entre lo que la sociedad espera de los medios desde el punto de vista normativo, y lo que los medios pueden otorgar en las condiciones en las que actúan. No obstante no todos los factores mencionados quedan de manifiesto en esta investigación y no queda claro cuál de ellos tiene más peso en la determinación final del producto. En 
última instancia el alcance de este trabajo es predominantemente de tipo exploratorio, más preocupado por describir el resultado textual de estas condiciones que la influencia concreta de las mismas.

Sería conveniente la realización de estudios empíricos específicos de cada factor para saber cómo se construye el engranaje completo de las representaciones periodísticas de la pobreza. Se concluye, sin embargo, que a pesar de las fuerzas que generan las insuficientes representaciones que medimos, de suyo inconscientes para los productores de las mismas, la verticalidad normativa y ética de los medios de referencia estudiados alcanza a contener el efecto estructural, organizacional e ideológico que se cierne sobre el periodismo al hablar de este tema. El corpus medido y analizado no deja duda de la presencia de asimetrías sistemáticas de poder que disminuyen la importancia pública del tema de la pobreza y lo hacen dependiente a la presencia de las élites.

Se constata asimismo que la prensa no cumple la expectativa de hacer que la sociedad se conozca más a sí misma y a sus problemas más acuciantes. Tenemos aquí elementos para suponer la contribución de estas representaciones al status quo. Pero no podemos dejar de lado que de manera contradictoria no tenemos datos que evidencien una explotación ramplona o siquiera sutil del tema y que los parámetros mínimos de seriedad profesional son constantes. La cotidiana contradicción entre lo que el periodismo quiere y puede ser, encuentra en este particular caso estudiado, un inestimable ejemplo. Lamentablemente, la prensa mexicana analizada encuentra también en el tratamiento de la pobreza una sinécdoque de sus limitaciones.

\section{FUENTES REFERENCIALES}

Berger, G. (2003, 25-27 June). A half-told story: developing a research agenda into representation of poverty in the South African news media. Paper presented at the Annual SACOMM conference, Durban.

Berger, P., \& Luckmann, T. (2005). La construcción social de la realidad. Buenos Aires: Amorrortu.

Bourdieu, P. (2000). Cosas dichas. Barcelona: Gedisa.

Coneval. (2009). Informe de evaluación de la política de desarrollo social en México [Electronic Version]. Retrieved 25 junio from http:// www.coneval.gob.mx/contenido/home/2509.pdf.

Couldry, N. (2000). The place of media power, pilgrims and witnesses of the media age. London: Routledge.

Echeverría, M. (2007). Representaciones periodísticas de la pobreza. Perspectivas teóricas para una agenda de investigación. En M. A. Rebeil (Ed.), XIV Anuario de investigación de la comunicación. 
México: Universidad Anáhuac Norte, UAM, UDEM, Unison, UVM, USB.

FSP. (2007). Pobreza: El desafío de (re) construir la noticia. Santiago: Alianza Comunicación y Pobreza.

Fundación para la Superación de la Pobreza, U. V. d. M. (2007). Pobreza: el desafío de (re) construir la noticia. Santiago: Alianza Comunicación y Pobreza.

Gallardo, L. R., \& Osorio, J. (2001). Los rostros de la pobreza. El debate (vol. 1). México: Iteso, Universidad Iberoamericana, Limusa.

Gamson, W. A., Croteau, D., Hoynes, W., \& Sasson, T. (1992). Media images and the social construction of reality. Annual Review of Soci$\operatorname{ology}(18), 373-393$.

Golding, P. (1999). Thinking the unthinkable. Welfare reform and the media. En B. Franklin (Ed.), Social policy media \& misrepresentation. Florence: Routledge, en Franklin (Ed.), Social policy media \& misrepresentation. Florence: Routledge.

Golding, P., \& Murdock, R. (2000). Culture, communications and political economy. In J. Curran \& M. Gurevitch (Eds.), Mass media and society. London: Arnold.

Gordon, S. (1997). Pobreza y patrones de exclusión social en México. México: Instituto Internacional de Estudios Laborales.

Hernández, S. (2003). Un acercamiento a la nota roja: `La inclusión y exclusión de las clases vulnerables. Revista Electrónica Sala de Prensa, 2(45).

Karam, T. (2003). Fotografía periodística, discurso visual y derechos humanos en la prensa de la Ciudad de México [Electronic Version]. Razón y Palabra. Retrieved 8-09-09 from http://www.razonypalabra.org.mx/anteriores/n36/ tkaram.html. Kendall, D. A. (2005). Framing Class. Media representations of wealth and poverty in America. Oxford: Rowman and Littlefield.

Leñero, O. (1995). Los pobres en México: Su promoción. México: Instituto Mexicano de Estudios Sociales, A.C.

Maigret, É. (2005). Sociología de la comunicación y de los medios. Bogotá: Paidos.

Martín Serrano, M. (1994). La producción social de la comunicación (Segunda edición ed.). México: Alianza Editorial.

Murdock, G., \& Golding, P. (1982). Capitalismo, comunicaciones y relaciones de clase. En J. Curran, M. Gurevitch \& J. Woollacot (Eds.), Sociedad y comunicación de masas. México: FCE.

Ortega Villa, L. M. (2007). Uso de métodos cuantitativos y cualitativos en el estudio del consumo de bienes culturales en sectores populares de Mexicali, B.C. Estudios Fronterizos, 8(16), 43-63. 
Rey, G. (2004). La pobreza en las páginas de El Tiempo. Bogotá: El Tiempo, PNUD.

Richardson, J. A. (2007). Analysing newpapers. An approach from critical discourse analysis. New York: Palgrave McMillan.

Riffe, D., Lacy, S., \& Fico, F. (1998). Analyzing media messages: Using quantitative content analysis in research. New Jersey: Lawrence Erlbaum. Riquer, F. (2002). Mujer, género y pobreza: Estado de la discusión en los noventa. In L. R. Gallardo \& J. Osorio (Eds.), Los rostros de la pobreza. El debate (vol. II). México: Iteso, Universidad Iberoamericana, Limusa.

Sampedro, V., \& Codeiro, T. (2001). Violencia y medios, una cuestion de responsabilidad social. BAHIA ANÁLISE \& DADOS, 11(1), 17-22.

Tablante, L. (2006, junio 2006). La pobreza como objeto mediático en Venezuela. Paper presented at the XII Encuentro Latinoamericano de Facultades de Comunicación Social, Bogotá, Colombia.

Tuchman, G. A. (1983). La producción de la noticia. Estudio sobre la construcción de la realidad. Barcelona: Gustavo Gili.

Vasilachis, I. (2003). Pobres, pobreza, identidad y representaciones sociales. Barcelona: Gedisa.

Vázquez, N. I. (2008). La violencia mediática: Un estudio de caso. Convergencia, 15(47), 103-125.

Wayne, M. (2003). Marxism and media studies, Key concepts and contemporary trends (First ed.). London: Pluto Press. 